\title{
Astrocytes enhance the tolerance of rat cortical neurons to glutamate excitotoxicity
}

\author{
LI-NAN ZHANG ${ }^{*}$, QI WANG ${ }^{*}$, XIAO-HUI XIAN, JIE QI, LI-ZHE LIU and WEN-BIN LI \\ Department of Pathophysiology, Hebei Medical University, Shijiazhuang, Hebei 050017, P.R. China
}

Received March 24, 2017; Accepted October 31, 2018

DOI: $10.3892 / \mathrm{mmr} .2018 .9799$

\begin{abstract}
Glutamate excitotoxicity is responsible for neuronal death in acute neurological disorders, including stroke, trauma and neurodegenerative diseases. Astrocytes are the main cells for the removal of glutamate in the synaptic cleft and may affect the tolerance of neurons to the glutamate excitotoxicity. Therefore, the present study aimed to investigate the tolerance of rat cortical neurons to glutamate excitotoxicity in the presence and absence of astrocytes. Rat cortical neurons in the presence or absence of astrocytes were exposed to different concentrations of glutamate $(10-2,000 \mu \mathrm{M})$ and $10 \mu \mathrm{M}$ glycine for different incubation periods. After $24 \mathrm{~h}$, the Cell Counting kit-8 (CCK-8) assay was used to measure the cytotoxicity to neurons in the presence or absence of astrocytes. According to the results, in the absence of astrocytes, glutamate induced a concentration-dependent decrease of neuronal survival rate compared with the control rat cortical neurons, and the neurotoxic half-maximal inhibitory concentration $\left(\mathrm{IC}_{50}\right)$ at 15 , 30 and 60 min was $364.5,258.5$ and $138.3 \mu \mathrm{M}$, respectively. Furthermore, in the presence of astrocytes, glutamate induced a concentration-dependent decrease of neuronal survival rate compared with the control rat cortical neurons, and the neurotoxic $\mathrm{IC}_{50}$ at 15,30 and 60 min was $1,935,932.8$ and $789.3 \mu \mathrm{M}$, respectively. However, astrocytic toxicity was not observed when the rat cortical astrocytes alone were exposed to different concentrations of glutamate (500, 1,000 and 2,000 $\mu \mathrm{M}$ ) for 6, 12 and $24 \mathrm{~h}$. In conclusion, the glutamate-induced neurotoxic $\mathrm{IC}_{50}$ values at 15, 30 and $60 \mathrm{~min}$ were respectively higher in the presence of astrocytes as compared with those in the absence of astrocytes, suggesting that astrocytes can protect against rat cortical neuronal acute damage induced by glutamate.
\end{abstract}

Correspondence to: Dr Wen-Bin Li, Department of Pathophysiology, Hebei Medical University, 361 Zhongshan East Road, Shijiazhuang, Hebei 050017, P.R. China

E-mail: liwbsjz@163.com

${ }^{*}$ Contributed equally

Key words: glutamate, excitotoxicity, tolerance, neuron-astrocyte co-culture, cortical neurons

\section{Introduction}

A number of previous studies have reported an in vivo ambient glutamate concentration as high as $1-4 \mu \mathrm{M}$ (1-3). Given that the $50 \%$ effective concentration $\left(\mathrm{EC}_{50}\right)$ of the $\mathrm{N}$-methyl-D-aspartate (NMDA) receptor for glutamate was $\sim 2 \mu \mathrm{M}$ (4), this concentration range would have significant effects on neuronal excitability. However, opposing data indicated that the ambient glutamate concentration was lower, within the nanomolar range, which was in better agreement with the theoretical minimum concentration of glutamate $(2 \mathrm{nM})(5)$.

Treatment with two different concentrations of glutamate (175 and $250 \mu \mathrm{M}$ ) for $1 \mathrm{~h}$ led to different outcomes in rat cortical cells, and cultures treated with $250 \mu \mathrm{M}$ glutamate suffered a loss in overall activity that was not observed in cultures treated with $175 \mu \mathrm{M}$ glutamate in rat cortical cells, serving as a model of traumatic brain injury (6). Loss of calcium homeostasis is a key mediator of glutamate-induced cell death, which is involved in Alzheimer's disease (7) and other age-associated neurodegenerative conditions, such as oxidative stress (8) and cellular energy deficits (9). It has been reported that glutamate $(8 \mathrm{mM})$ treatment alone caused a significant increase $(50 \%)$ in cell death compared with the control group in the HT22 mouse hippocampal cell line, whereas glutamate $(1 \mathrm{mM})$ treatment alone induced one fold increase $(50 \%)$ in cell death compared with the control group in primary cultures of rat cortical neuronal cells (10), where astrocytes could be involved in the neuroprotective effect. Certain studies have demonstrated that astrocytes expressing glutamic acid decarboxylase 67 (GAD67) protected primary neurons from the toxicity of exposure to $300 \mu \mathrm{M}$ glutamate for $10 \mathrm{~min}$ (11) or from serum glutamate (12). Specific compounds, such as pyruvate, $\beta$-amyloid and ceftriaxone, act to protect neurons from damage during brain ischemia via astrocytes (7-9). However, co-cultures of neurons and astrocytes have been reported to increase neuronal sensitivity to glutamate treatment for $24 \mathrm{~h}$ (13).

According to these controversial results, the aim of the present study was to investigate glutamate-induced rat cortical neuronal toxicity in the presence or absence of astrocytes. The study also further explored the protection of neurons by astrocytes.

\section{Materials and methods}

Materials. Dulbecco's modified Eagle's medium (DMEM)/F12, neurobasal medium, B27 supplement and 
fetal bovine serum (FBS) were purchased from Thermo Fisher Scientific, Inc. (Gibco; Waltham, MA, USA). Trypsin was purchased from Lonza Bioscience (Walkersville, MD, USA). Penicillin and streptomycin were purchased from Biological Industries (Beit Haemek, Israel). Poly-D-lysine, monosodium glutamate, DAPI and DNase were obtained from Sigma-Aldrich (Merck KGaA, Darmstadt, Germany). Mouse monoclonal anti-neuronal nuclei (NeuN; GTX30773) antibody as a neuronal marker was purchased from GeneTex, Inc. (Irvine, CA, USA), and mouse monoclonal anti-glial fibrillary acidic protein (GFAP) as astrocyte marker was purchased from BD Biosciences, Franklin Lakes, NJ, USA (556328). 5-Fluorouracil and uridine were from TCI Development Co., Ltd. (Shanghai, China). Cell Counting kit-8 (CCK-8) was purchased from Zoman Biotechnology Co., Ltd. (Beijing, China), while papain was obtained from Acros Organics (Belgium). Cell culture plates, flasks and inserts were purchased from Corning, Inc. (Corning, NY, USA).

Animals. Newborn Wistar rats (0-1 day) were obtained to culture cortical neurons, and newborn Wistar newborn rats (1-3 days) to culture cortical astrocytes (Hebei Medical University, Shijiazhuang, China). The pregnant and neonatal rats $(5.5 \sim 10 \mathrm{~g})$ were housed with standard chow and water ad libitum in an ambient temperature of $22 \pm 2^{\circ} \mathrm{C}$ and kept under a 12/12 h light/dark cycle with the lights on at 07:00 a.m. All animal care and experimental procedures were performed in accordance with approved guidelines of the National Institutes of Health for the Care and Use of Laboratory Animals, and the guidelines were approved by the Committee of Ethics on Animal Experiments of Hebei Medical University. All efforts were made to minimize suffering and the number of animals was used in the study.

Primary rat cortical neuronal culture. Primary cultures of cortical neurons from newborn Wistar rats (0-1 day) were obtained as described previously (14). Briefly, the brains of newborn Wistar rats (0-1 day) were removed subsequent to decapitation, and the meninges were stripped away. The cortices of these brains were dissected in ice-cold DMEM-F12 basal culture media supplemented with $26 \%$ glucose. The tissues were treated with $2 \mathrm{mg} / \mathrm{ml}$ papain and $42 \mu \mathrm{g} / \mathrm{ml}$ DNase for $30 \mathrm{~min}$ in $37^{\circ} \mathrm{C}$ incubator, and then gently dissociated by trituration in neuronal culture media $(97 \%$ neurobasal medium supplemented with $2 \% \mathrm{~B} 27,100 \mathrm{U} / \mathrm{ml}$ penicillin and $100 \mu \mathrm{g} / \mathrm{ml}$ streptomycin). The digestion was terminated by FBS at room temperature, and the cell suspension was filtered prior to centrifugation ( $447 \mathrm{x}$ g for $10 \mathrm{~min}$ room temperature). Cells were centrifuged three times, washed with Hanks' balanced salt solution (with $1.3 \mathrm{mM}$ calcium and $0.5 \mathrm{mM}$ magnesium) and finally resuspended in neurobasal medium. Cells were then seeded on $1 \mu \mathrm{g} / \mathrm{ml}$ poly-D-lysine-coated 24 -well plates at a density of $7 \times 10^{5}$ cells/well and maintained in neuronal culture media in a humidified atmosphere with $5 \% \mathrm{CO}_{2}$ at $37^{\circ} \mathrm{C}$. Culture medium was replaced every 2-3 days to minimize culture debris. Pure neuronal cultures were obtained by addition of $1 \mu \mathrm{mol} / \mathrm{L} 5$-fluorouracil and uridine in the culture at day 3 , which was refreshed by neuronal culture media 2 days later. After 9-11 days, the purity of neurons was identified by $\mathrm{NeuN}$ and DAPI fluorescent staining.
Primary rat cortical astrocyte culture. Cerebral cortical astrocytes were prepared as previously described, with slight modification (15). Briefly, cultured cortices were obtained from Wistar newborn rats (1-3 days) and passed through a nylon sieve (75- $\mu \mathrm{m}$ pore size) into DMEM/F12 supplemented with $10 \% \mathrm{FBS}, 100 \mathrm{U} / \mathrm{ml}$ penicillin and $100 \mu \mathrm{g} / \mathrm{ml}$ streptomycin. The cell suspension was seeded in poly-D-lysine-coated flasks at $37^{\circ} \mathrm{C}$ in a humidified atmosphere with $5 \% \mathrm{CO}_{2}$. The medium was refreshed the following day and changed every two days until the cells covered $80-90 \%$ of the culture flask. At 9-11 days, these primary cultures were purified by a physical method to eliminate non-astrocytic cells. The flasks were shaken inside an incubator for 12-18 h, detached cells were eliminated, and the medium was renewed. Residuary cells were digested with $0.25 \%$ trypsin, seeded into 24 -well plates $\left(8 \times 10^{5}\right.$ cells/plate) and maintained in DMEM/F12 culture media in a humidified atmosphere with $5 \% \mathrm{CO}_{2}$ at $37^{\circ} \mathrm{C}$. Culture medium was replaced every 2-3 days to minimize culture debris. Experiments were usually conducted on the third day.

Primary rat cortical co-culture of neurons and astrocytes. Neuron-astrocyte co-cultures were generated by plating pure neurons in the wells of a 6 -well plate $\left(1 \times 10^{6}\right.$ cells/well $)$ and pure astrocytes $\left(8 \times 10^{5}\right.$ cells/well) in the culture strip inserts of the plates with neuronal culture media for 2 days. Samples were kept in an incubator with a humidified atmosphere and $5 \% \mathrm{CO}_{2}$ at $37^{\circ} \mathrm{C}$. An Olympus inverted phase contrast microscope (Olympus Corporation, Tokyo, Japan) was used to capture images of cell density, morphology and spreading on the multilayer surfaces.

Immunofluorescence analysis. Cortical neurons and astrocytes were rinsed in PBS, and fixed with $4 \%$ paraformaldehyde for $>2 \mathrm{~h}$. Subsequent to washing in PBS, cells were incubated for 20 min in $0.4 \%$ Triton $\mathrm{X}-100$ in $\mathrm{PBS}$ at $37^{\circ} \mathrm{C}$, further washed three times with PBS, blocked with $10 \%$ goat serum at $37^{\circ} \mathrm{C}$ for $1 \mathrm{~h}$ and then washed for a further three times with PBS. Next, neurons were incubated overnight at $4^{\circ} \mathrm{C}$ with anti-neuron-specific nuclear protein NeuN monoclonal antibody (dilution, 1:150 in PBS), followed by five washes with PBS for 5 min each. The neurons were subsequently incubated with a 488-conjugated goat anti-mouse secondary antibody (072031806; Kirkegaard \& Perry Laboratories Inc., Gaithersburg, MD, USA; dilution, 1:200 in PBS) for $1 \mathrm{~h}$ at $37^{\circ} \mathrm{C}$ and washed for a further five times in PBS. Similarly, astrocytes were incubated with mouse anti-GFAP monoclonal antibody (BD Biosciences; dilution, 1:200 in 10\% goat serum), washed five times in PBS, and then incubated with a KPL 488-conjugated goat anti-mouse secondary antibody (Gaithersburg, MD, USA; dilution 1:200 in PBS) for $1 \mathrm{~h}$ at $37^{\circ} \mathrm{C}$, followed by further washing for five times in PBS. Subsequently, neurons or astrocytes were incubated with $0.005 \%$ DAPI for $10 \mathrm{~min}$ at room temperature and further washed five times in PBS. Images were collected on a Nikon Eclipse Ti-E inverted fluorescence microscope (Nikon Corporation, Tokyo, Japan), and then processed and visualized using NIS-Elements D (version 4.50; Nikon Corporation) to assess NeuN/DAPI and GFAP/DAPI staining. High-resolution images were created using Photoshop CS2 (Adobe, Inc., Mountain View, CA, USA). 
A

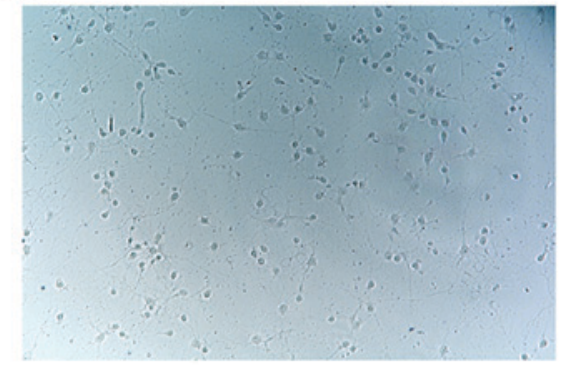

C

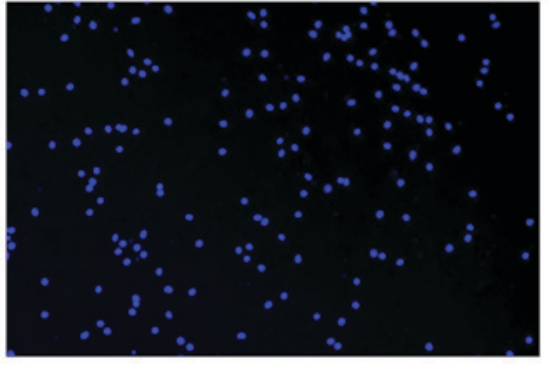

B

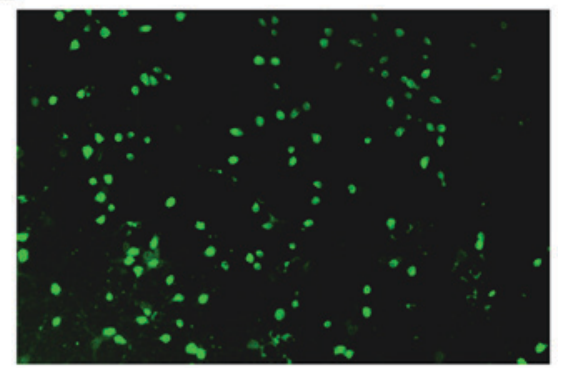

D

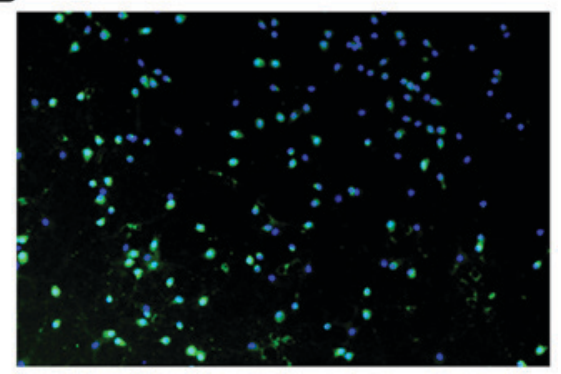

Figure 1. Immunofluorescence characterization of neurons (magnification, x200). (A) Bright-field micrographs of neurons on the culture plate surface. (B) NeuN, (C) DAPI and (D) combined NeuN and DAPI staining of neurons on the culture plate surface. NeuN, neuronal nuclei.

Glutamate-induced exitotoxicity in rat cortical neurons and/or astrocytes. Monosodium glutamate was dissoluted with water, and the stock solution of 2,000 $\mathrm{mM}$ was stored at $-20^{\circ} \mathrm{C}$. Rat cortical neurons alone were exposed to 10,25 , $50,100,200,500$ and 1,000 $\mu \mathrm{M}$ glutamate and $10 \mu \mathrm{M}$ glycine (there is glycine combining site in glutamate NMDA receptors, and glutamate-induced effect can be increased in the presence of glycine) for 15, 30 and $60 \mathrm{~min}$ in $\mathrm{Mg}^{2+}$-free Locke's buffer (154 mM NaCl, 5.6 mM KCl, 3.6 mM NaHCO $3,1.3 \mathrm{mM}$ $\mathrm{CaCl}_{2}, 5.6 \mathrm{mM}$ D-glucose and 5 mM HEPES; pH 7.4) (16). In addition, co-cultures of rat cortical neurons and astrocytes were exposed to $10,100,200,500,1,000$ and 2,000 $\mu \mathrm{M}$ glutamate and $10 \mu \mathrm{M}$ glycine for 15,30 and $60 \mathrm{~min}$ in $\mathrm{Mg}^{2+}$-free Locke's buffer. Rat cortical astrocytes alone were exposed to $500,1,000$ and $2,000 \mu \mathrm{M}$ glutamate for 6,12 and $24 \mathrm{~h}$ in DMEM-F12 basal medium. Next, glutamate was washed out thoroughly, and replaced with neurobasal culture media or DMEM-F12 complete medium in a humidified atmosphere with $5 \% \mathrm{CO}_{2}$ at $37^{\circ} \mathrm{C}$. After $24 \mathrm{~h}$, a CCK-8 assay was used to assess the cell viability.

Cell viability. The cell viability of cultured rat cortical neurons, astrocytes, and the co-culture of neurons and astrocytes was measured $24 \mathrm{~h}$ after glutamate treatment by CCK- 8 assay. Briefly, CCK-8 solution (10\% final concentration) was added to cultured neurons and/or astrocytes, and incubated for $4 \mathrm{~h}$. The optical densityof each sample was measured using BioTek Synergy 2 (BioTek Instruments, Inc., Winooski, VT, USA) at $450 \mathrm{~nm}$. Quantitative dose-toxicity examination was performed in the rat cortical neurons alone, astrocytes alone and the co-culture of neurons and astrocytes, and the half-maximal inhibitory concentration $\left(\mathrm{IC}_{50}\right)$ was measured.

Statistical analysis. All data are expressed as the mean \pm standard error of the mean. Each $n$ value represents data from one culture well. Unless otherwise indicated, an $\mathrm{n}$ value of 8-12 culture wells was used for each group in each experiment, with repetitions conducted using at least 3 independent dissections for each experiment. Differences between group means were analyzed with one-way analysis of variance, followed by the Bonferroni posthoc test. The differences were considered as statistically significant when the P-value was $<0.05$. Nonlinear regression analysis and $\mathrm{IC}_{50}$ estimates for dose-response data were performed with GraphPad Prism software (version 6.0; GraphPad Software, Inc., La Jolla, CA, USA).

\section{Results}

Immunofluorescence characterization of neurons and astrocytes. NeuN-immunofluorescence analysis indicated that the purity of primary neurons was $>95 \%$, and dispersed neurons with rare clumping were detected (Fig. 1). In addition, GFAP-immunofluorescence analysis revealed that the purity of primary flat polygonal astrocytes was $>95 \%$ (Fig. 2; data not shown).

Glutamate-induced exitotoxicity of rat cortical neurons in the absence of astrocytes. In the absence of astrocytes, the exposure of rat cortical neurons to various glutamate concentrations $(10,25,50,100,200,500$ and 1,000 $\mu \mathrm{M})$ and $10 \mu \mathrm{M}$ glycine for 15,30 or $60 \mathrm{~min}$, respectively, resulted in a concentration-dependent decrease of the neuronal survival rate compared with the control group $\left(\mathrm{Mg}^{2+}\right.$-free Locke's buffer without glutamate and glycine). As shown in Fig. 3A, treatment with $10 \mu \mathrm{M}$ glutamate and $10 \mu \mathrm{M}$ glycine had no evident effect on neuronal survival at the three time points, while exposure to $25 \mu \mathrm{M}$ glutamate and $10 \mu \mathrm{M}$ glycine had a significant effect on neuronal survival, reducing the survival rate to approximately $50-70 \%$ of the control value. The neuronal survival rates of glutamate $(25-1,000 \mu \mathrm{M})$ to control were reduced in a concentration-dependent manner, 
A

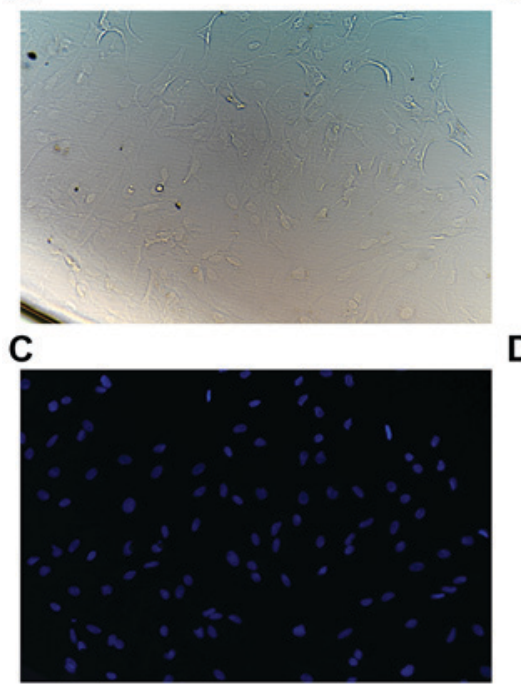

B

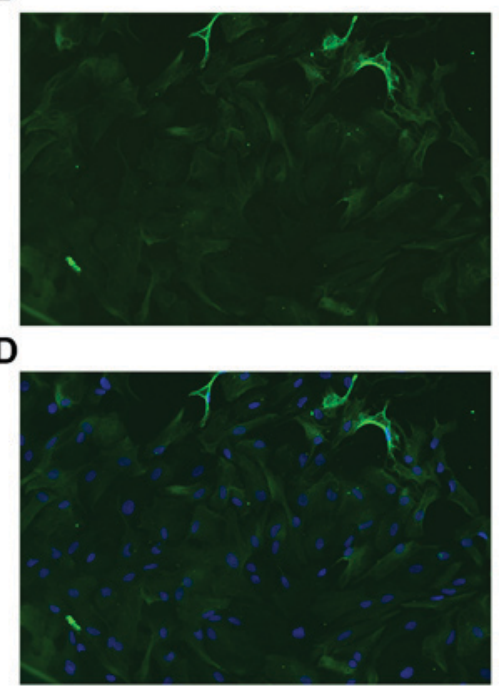

Figure 2. Immunocytochemical characterization of astrocytes (magnification, x200). (A) Bright-field micrographs of astrocytes on the coverslip. (B) GFAP, (C) DAPI and (D) combined GFAP and DAPI staining of astrocytes on the coverslip. GFAP, glial fibrillary acidic protein.

but no significant difference was observed between different glutamate concentrations (Fig. 3A). Furthermore, the $\mathrm{IC}_{50}$ of the survival rate in neurons exposed to glutamate for 15,30 and 60 min compared with the control was 364.5, 258.5 and $138.3 \mu \mathrm{M}$, respectively.

Glutamate-induced exitotoxicity of rat cortical astrocytes alone. Astrocytes extracted from rat cerebral cortex were exposed to different concentrations of glutamate for 6, 12 and $24 \mathrm{~h}$. The astrocytic survival rates following expose to glutamate $(500,1,000$ and $2,000 \mu \mathrm{M})$ were not markedly changed compared with the control group.

Glutamate-induced exitotoxicity of rat cortical neurons in the presence of astrocytes. The exposure of rat cortical neurons to different glutamate concentrations $(10,25,50,100,200$, $500,1,000$ and $2,000 \mu \mathrm{M}$ ) and $10 \mu \mathrm{M}$ glycine in the presence of astrocytes for 15,30 or $60 \mathrm{~min}$, respectively, resulted in a concentration-dependent decrease of the neuronal survival rate compared with the control group. As shown in Fig. 3B, treatment with $10 \mu \mathrm{M}$ glutamate and $10 \mu \mathrm{M}$ glycine had no evident effect on neuronal survival at the three incubation times. By contrast, treatment with $25 \mu \mathrm{M}$ glutamate and $10 \mu \mathrm{M}$ glycine resulted in an evident tendency of decreased neuronal survival, and the survival rate was reduced to approximately $70-80 \%$ of that of the control group, with a significant statistical difference observed at 30 and $60 \mathrm{~min}$ $(\mathrm{P}<0.01)$. In addition, the $\mathrm{IC}_{50}$ of the neuronal survival rate in the neuron-astrocyte co-culture exposed to glutamate for 15 , 30 and 60 min compared with the control was 1,935, 932.8 and $789.3 \mu \mathrm{M}$, respectively (Fig. 3B). In Fig. 4, the survival rate of neurons following different glutamate concentrations at different time periods was compared in neuron alone culture and neuron-astrocyte coculture. As demonstrated in Fig. 4A, the survival rate of treatment with 50, 100 and $200 \mu \mathrm{M}$ glutamate and $10 \mu \mathrm{M}$ glycine for $15 \mathrm{~min}$ in neuron-astrocyte coculture was significantly increased compared with that in neuron alone culture, while the survival rates of treatment with
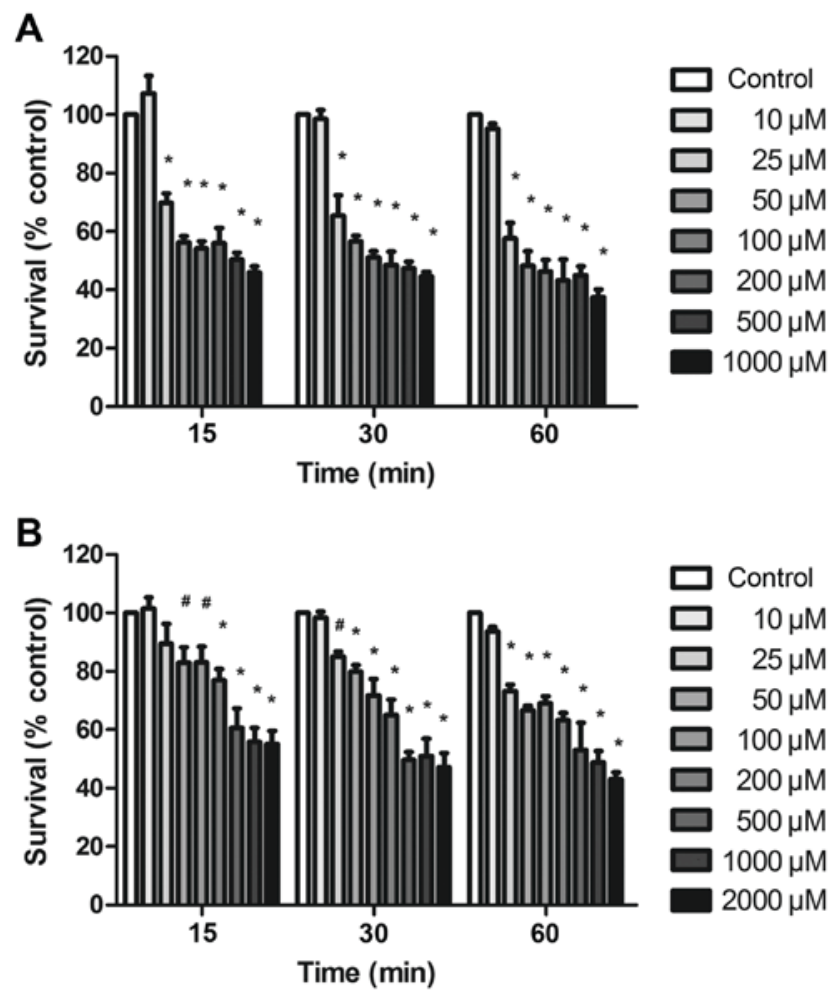

Figure 3. Dose-dependent survival rate of neurons in the (A) absence or (B) presence of astrocytes following treatment with different glutamate concentrations at different time periods. Student's t-test was conducted to determine statistically significant differences in the results. ${ }^{\#} \mathrm{P}<0.05$ and ${ }^{*} \mathrm{P}<0.01$ vs. control group $(100 \%)$.

25, 50, 100 and $200 \mu \mathrm{M}$ glutamate and $10 \mu \mathrm{M}$ glycine for 30 and $60 \mathrm{~min}$ in neuron-astrocyte coculture was significantly increased compared with that in neuron alone culture in Fig. 4B and C. Student's t-test was conducted to determine statistically significant differences in the results: ${ }^{\#} \mathrm{P}<0.05$ and ${ }^{*} \mathrm{P}<0.01$ vs. the survival rate of neurons in neuron alone culture. Furthermore, changes in the morphology of rat cortical neurons were observed at 15,30 and 60 min following 
A

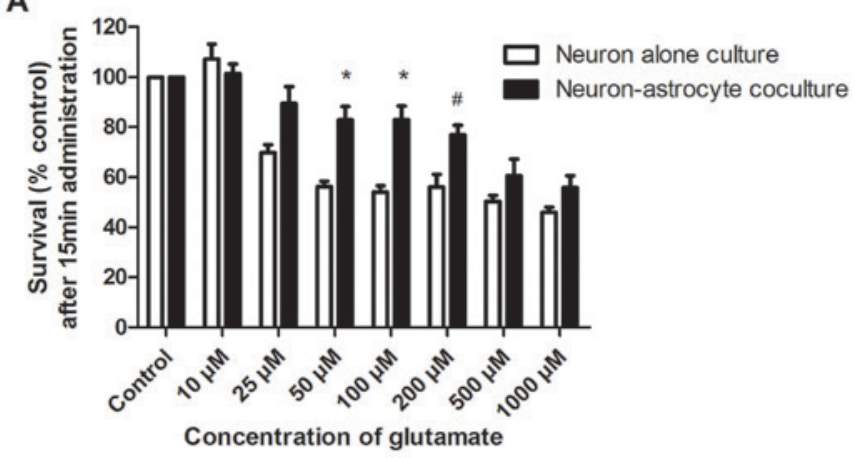

B

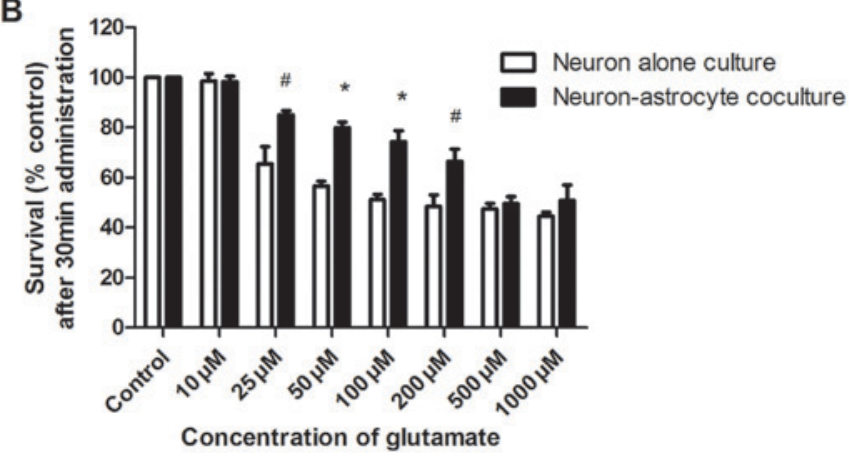

C

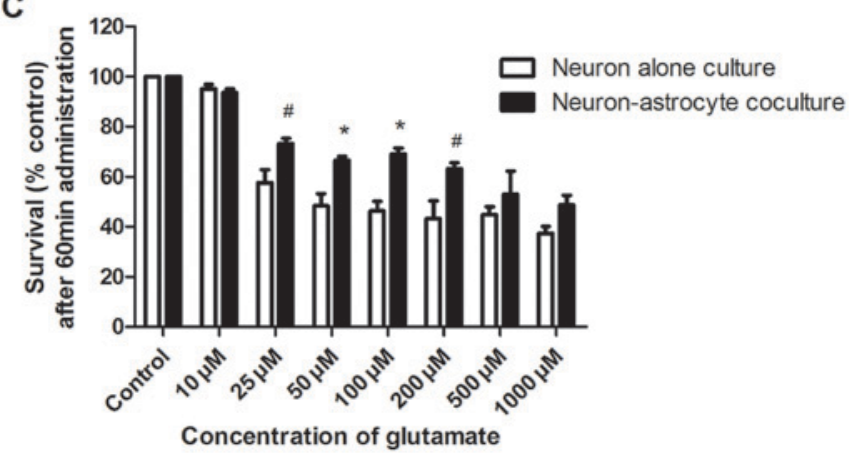

Figure 4. The comparison of survival rate of neurons in neuron alone culture and neuron-astrocyte coculture following treatment with different glutamate concentrations respectively at different time periods: (A) $15 \mathrm{~min}$, (B) $30 \mathrm{~min}$ and (C) $60 \mathrm{~min}$. Student's t-test was conducted to determine statistically significant differences in the results. ${ }^{\#} \mathrm{P}<0.05$ and ${ }^{*} \mathrm{P}<0.01$ vs. the survival rate of neurons in neuron alone culture.

glutamate (200 and 1,000 $\mu \mathrm{M})$ exposure in the presence of astrocytes as compared with the control group (Fig. 5), proving the concentration-dependent neurotoxicity of glutamate in the rat cortical co-culture of neurons and astrocytes. All the above results suggested that astrocytes enhanced the tolerance of rat cortical neurons to glutamate excitotoxicity.

\section{Discussion}

In the present study, glutamate exposure of neurons isolated from the cerebral cortex of newborn Wistar rats (0-1 day) in the absence of astrocytes for 15,30 and 60 min resulted in a concentration-dependent neurotoxicity $\left(\mathrm{IC}_{50}=364.5,258.5\right.$ and $138.3 \mu \mathrm{M}$, respectively). These results were consistent with previously reported findings that approximately $20 \pm 3 \%$ primary hippocampal neurons of postnatal Wistar rats (0-1 day) were destroyed in control cultures, while $40-50 \%$ neurons were destroyed in $0.5 \mathrm{mM} \mathrm{L}$-glutamate cultures for $30 \mathrm{~min}$ (17). In addition, in cultures exposed to $1 \mathrm{mM}$ L-glutamate, up to $60 \%$ of the neurons died during this time period of $30 \mathrm{~min}$ depending on the concentration of glutamate (17). However, the results of the present study were inconsistent with another study that addition of glutamate $(50 \mu \mathrm{M})$ to primary cortical neurons obtained from rat embryos [embryonic day(E) 16-18] for $1 \mathrm{~h}$ resulted in a living rate of neurons of $\sim 20 \%$ in contrast to the control group (18). Furthermore, the apoptotic-like death of cultured rat cortical neurons from 17-day-old Sprague-Dawley rat embryos exposed to $50 \mu \mathrm{M}$ glutamate for 15 min was up to $\sim 80 \%$ (19). Therefore, the difference in glutamate-induced neurotoxicity between the present study and previous studies may be a result of the culture being obtained from newborn or fetal rats, and the neuron culture of the fetal rats may be more sensitive to exposure to glutamate as compared with that of the newborn rats.

Glutamate $(10 \mathrm{mM})$ applied to the mouse hippocampal HT2 2 cell line for $24 \mathrm{~h}$ has been reported to reduce the neuronal cell survival by $>80 \%$ (20). HT22 cells do not express functional glutamate receptors; therefore, glutamate toxicity was mediated by inhibiting the cystine uptake (21). Furthermore, another study demonstrated that cellular death was observed at $\sim 6 \mathrm{~h}$ after initiation of $1 \mathrm{mM}$ glutamate exposure in primary cortical neurons isolated from 18-day-old pregnant Sprague-Dawley rats, and after $\sim 8 \mathrm{~h}$ of exposure to 7-8 mM glutamate in HT22 cells (10). In addition, brief exposure to glutamate was found to produce morphological changes in mature (14-24 days in vitro) cortical neurons from fetal mouse neocortex, beginning as quickly as $90 \mathrm{sec}$ after exposure, followed by widespread neuronal degeneration over the next hours, and a quantitative dose-toxicity study suggested an $\mathrm{EC}_{50}$ of 50-100 $\mu \mathrm{M}$ for a 5-min exposure to glutamate (22). The reliable neurotoxicity produced by exposure of cell cultures to $0.5 \mathrm{mM}$ glutamate for $5 \mathrm{~min}$ was only observed with mature cultures, whereas immature (5-7 days in vitro) neurons and glia were unaffected by such glutamate exposure, and partial effects were observed with cultures of intermediate maturity (22). Therefore, the difference of glutamate-induced neurotoxicity between the present study and previous studies may also be due to differences in primary neuron cultures and nervous cell lines, genera of animals or degrees of maturity, which may depend on the distribution density of glutamate receptors. Nervous cell lines that do not express functional glutamate receptors may be more resistant to glutamate-induced neurotoxicityin comparison with primary neuronal culture, while the distribution of glutamate receptors of immature (5-7 days in vitro) culture neurons may be less than that of mature (14-24 days in vitro) culture neurons.

In the present study, astrocytic toxicity was not observed when astrocytes isolated from the rat cerebral cortex were exposed to different concentrations of glutamate $(500,1,000$ and $2,000 \mu \mathrm{M}$ ) for 6,12 and $24 \mathrm{~h}$ (data not shown). This is consistent with previous observations suggesting that cerebral astrocytes isolated from the cerebral cortex of 1-3-day Wistar newborn rats were resistant to injury by glutamate even in the presence of the uptake inhibitor (23). The morphology of astrocytes from fetal mouse neocortex appeared to be completely unaffected by $0.5 \mathrm{mM}$ glutamate for $5 \mathrm{~min}$, and no evidence of direct 

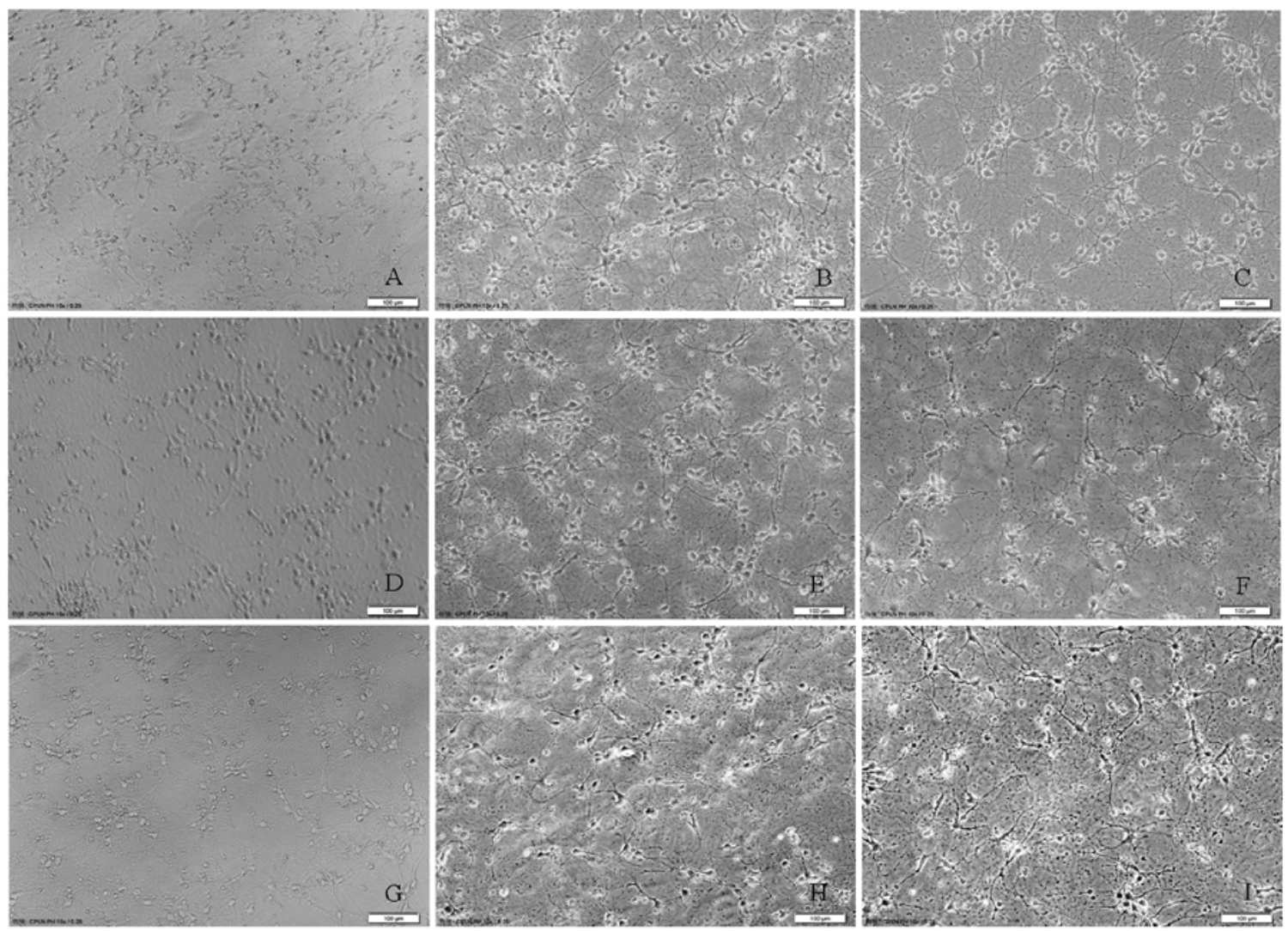

Figure 5. Morphological characteristics of rat primary neurons following exposure to different concentrations of glutamate for 15,30 or 60 min in the presence of astrocytes (magnification, x100). Neurons co-cultured with astrocytes were exposed to (A) 0 , (B) 200 and (C) $1,000 \mu \mathrm{M}$ glutamate for 15 min. Neurons co-cultured with astrocytes were exposed to (D) 0 , (E) 200 and (F) 1,000 $\mu \mathrm{M}$ glutamate for 30 min. Neurons co-cultured with astrocytes were exposed to (G) $0,(\mathrm{H}) 200$ and (I) $1,000 \mu \mathrm{M}$ glutamate for $60 \mathrm{~min}$. Scale bar,100 $\mu \mathrm{m}$.

glutamate gliotoxicity was detected on glial elements in mixed cortical cultures or in essentially pure glial cultures prepared from postnatal animals (22). Furthermore, another study reported that astrocytic toxicity was not induced by glutamate concentrations $<2 \mathrm{mM}$, and astrocytic death was not observed even after a 24-h exposure to up to $100 \mu \mathrm{M}$ glutamate in astrocytes alone or co-cultured with neurons (13). However, the glutamate gliotoxicity has been observed in pure glial cultures prepared from chick retinal cells (24). In vivo experiments also demonstrated the swelling of glia with glutamate neurotoxicity, but the gliotoxicity was not observed upon treatment with low doses of glutamate (25). Therefore, combining these results with the findings of the present study, whether glutamate can induce gliotoxicity may depend on various factors, including the genera, maturity degree, concentration, and in vivo or in vitro culture among others, although this should be further explored.

The present study assessed the neuronal cytotoxic effect of glutamate on rat cortical co-culture of neurons and astrocytes, which were incubated with different concentrations of glutamate (including 10, 25, 50, 100, 200, 500, 1,000 and 2,000 $\mu \mathrm{M}$ ) for 15,30 and $60 \mathrm{~min}$. For each concentration of glutamate, $10 \mu \mathrm{M}$ glycine was added to the medium, followed by assessment of cell viability using the CCK-8 assay subsequent to the incubation period. The $\mathrm{IC}_{50}$ values of the glutamate-induced neuronal survival rate of the co-culture of neurons and astrocytes for 15 , 30 and $60 \mathrm{~min}$ were respectively $1,935,932.8$ and $789.3 \mu \mathrm{M}$, indicating the time-dependent neurotoxicity of glutamate in the rat cortical co-culture of neurons and astrocytes. By contrast, the
$\mathrm{IC}_{50}$ values of the glutamate-induced neuronal survival rate in the neuron culture in the absence of astrocytes were 364.5, 258.5 and $138.3 \mu \mathrm{M}$ for 15,30 and $60 \mathrm{~min}$ incubation, respectively. Compared with the culture without astrocytes, the $\mathrm{IC}_{50}$ of the glutamate-induced neuronal survival rate in the presence of astrocytes was higher at each of the different incubation periods. These findings were consistent with the observations of a previous study, indicating that the $\mathrm{EC}_{50}$ of neuronal cytotoxicity in pure neuron cultures isolated from albino ICR mice at E15 following exposure to glutamate for $15 \mathrm{~min}$ was $\sim 5 \mu \mathrm{M}$, while that of neuron and astrocyte co-cultures was $\sim 100 \mu \mathrm{M}$ (13). The $\mathrm{EC}_{50}$ of glutamate-induced neurotoxicity $\left(\mathrm{EC}_{50}=200 \mu \mathrm{M}\right)$ in neuron-astrocyte co-cultures isolated from the embryonic (E17-19) and newborn rat cerebral cortex was greater compared with neuron-enriched cultures $\left(\mathrm{EC}_{50}=50 \mu \mathrm{M}\right)(23)$. Furthermore, the survival rates of treatment with 50,100 and $200 \mu \mathrm{M}$ glutamate and $10 \mu \mathrm{M}$ glycine for 15,30 and $60 \mathrm{~min}$ in neuron-astrocyte coculture were significantly increased respectively compared with that in neuron alone culture. However, the survival rates of treatment with 500 and $1,000 \mu \mathrm{M}$ glutamate and $10 \mu \mathrm{M}$ glycine for 15,30 and $60 \mathrm{~min}$ in neuron-astrocyte coculture exhibited a tendency to increase without significantly statistical differences, suggesting that astrocytes can enhance the tolerance of rat cortical neurons to glutamate excitotoxicity within a certain range.

Protection of $\mathrm{PC} 12$ cells against $\mathrm{H}_{2} \mathrm{O}_{2}$ or serum deprivation by co-culture with C8-GAD67 astrocytes has been reported in the presence of $1 \mathrm{mM}$ glutamate for $24 \mathrm{~h}$, while the primary 
cortical neuron survival was enhanced by co-culture with C8-GAD67 astrocytes, demonstrating that GAD-expressing astrocytes induced an increase of antioxidant activity, protecting neurons from various injuries (11). A previous study indicated that excitotoxic glutamate exposure $(500 \mu \mathrm{M}$ glutamate and $10 \mu \mathrm{M}$ glycine for $10 \mathrm{~min}$ ) by microperfusion in mixed primary cortical neuron cultures from embryonic (E16) rats resulted in approximate $20-30 \%$ neuronal loss after $1 \mathrm{~h}$ (26). However, this excitotoxic glutamate exposure resulted in $87.0 \pm 1.6 \%$ loss of cortical neurons after $24 \mathrm{~h}$. The addition of MK-801 to the incubation medium resulted in significant protection from the glutamate-induced neuronal loss after $24 \mathrm{~h}$, suggesting that chronic toxic reaction to glutamate exposure may be receptor-dependent (26). Another study reported that reliable neurotoxicity produced by exposure of cell cultures isolated from fetal mouse neocortex to $0.5 \mathrm{mM}$ glutamate for 5 min was only observed with mature cultures, while immature (5-7 days in vitro) neurons and glia were unaffected by exposure to $0.5 \mathrm{mM}$ glutamate for $5 \mathrm{~min}$, and partial effects were observed with cultures of intermediate maturity (22). Furthermore, the $\mathrm{EC}_{50}$ of neuronal cytotoxicity in mouse pure neuron cultures exposed to glutamate for $24 \mathrm{~h}$ was $\sim 50 \mu \mathrm{M}$, while that of mouse neuron-astrocyte co-cultures was $\sim 5 \mu \mathrm{M}$ (13). This indicated that neuronal cytotoxicity was significantly increased in the presence of astrocytes as compared with pure neuron cultures for all glutamate concentrations for $24 \mathrm{~h}$, and that astrocytes increased neuronal sensitivity to chronic glutamate exposure (24 h) as opposed to acute exposure (13).

Therefore, these aforementioned studies indicated that astrocytes can protect acute glutamate-induced neuronal toxicity possibly due to glutamate rapidly being taken up by astrocytes. However, astrocytes can also aggravate chronic glutamate-induced neuronal toxicity through increasing neuronal sensitivity to chronic glutamate exposure, demonstrating that chronic toxic reaction to glutamate exposure may be receptor-dependent. Furthermore, glutamate-induced neuronal toxicity may be affected by a variety of factors, including the maturity of neurons and glia, and immature cells may be insensitive to glutamate exposure. However, the roles and underlying mechanism of astrocyte-induced protection against neuronal glutamate excitotoxicity remains controversial, and further studies need to be performed.

In conclusion, glutamate induced concentration-dependent neurotoxicity in rat cortical neurons in the presence or absence of astrocytes. The $\mathrm{IC}_{50}$ of neuronal survival rate in the presence of astrocytes was higher in comparison with that in the absence of astrocytes, suggesting that astrocytes can enhance the tolerance of rat cortical neurons to glutamate excitotoxicity.

\section{Acknowledgements}

Not applicable.

\section{Funding}

The authors acknowledge the support received for this study from the Natural Science Foundationof China (grant no. NSFC81402886), the Natural Science Foundation of Hebei Province (grant no. H2016208071), and the Hebei
Province Hundred Excellent Innovative Talents Support Scheme (III) (grant no. SLRC2017044).

\section{Availability of data and materials}

The analyzed data sets generated during the present study are available from the corresponding author on reasonable request.

\section{Authors' contributions}

L-NZ and W-BL designed the study; L-NZ, QW and L-ZL performed the experiments; X-HX and JQ analyzed the data; and L-NZ wrote the manuscript.

\section{Ethics approval and consent to participate}

All experiments were performed with the approval of the Ethics Committee of Hebei Medical University.

\section{Patient consent for publication}

Not applicable.

\section{Competing interests}

The authors declare that they have no competing interests.

\section{References}

1. Lerma J,Herranz AS, Herreras O, Abraira V and Martín del Río R: In vivo determination of extracellular concentration of amino acids in the rat hippocampus. A method based on brain dialysis and computerized analysis. Brain Res 384: 145-155, 1986.

2. Baker DA, Xi ZX, Shen H, Swanson CJ and Kalivas PW: The origin and neuronal function of in vivo nonsynaptic glutamate. J Neurosci 22: 9134-9141, 2002.

3. Nyitrai G, Kékesi KA and Juhász G: Extracellular level of GABA and Glu: In vivo microdialysis-HPLC measurements. Curr Top Med Chem 6: 935-940, 2006.

4. Patneau DK and Mayer ML: Structure-activity relationships for amino acid transmitter candidates acting at N-methyl-D-aspartate and quisqualate receptors. J Neurosci 10: 2385-2399, 1990.

5. Levy LM, Attwell D, Hoover F, Ash JF, Bjørås M and Danbolt NC: Inducible expression of the GLT-1 glutamate transporter in a CHO cell line selected for low endogenous glutamate uptake. FEBS Lett 422: 339-342, 1998.

6. Kutzing MK, Luo V and Firestein BL: Measurement of synchronous activity by microelectrode arrays uncovers differential effects of sublethal and lethal glutamate concentrations on cortical neurons. Ann Biomed Eng 39: 2252-2262, 2011.

7. Zhang J, An S, Hu W, Teng M, Wang X, Qu Y, Liu Y, Yuan Y and Wang D: The neuroprotective properties of hericium erinaceus in glutamate-damaged differentiated PC12 cells and an Alzheimer's disease mouse model. Int J Mol Sci 17: E1810, 2016.

8. Cassano T, Pace L, Bedse G, Lavecchia AM, De Marco F Gaetani S and Serviddio G: Glutamate and mitochondria: Two prominent players in the oxidative stress-induced neurodegeneration. Curr Alzheimer Res 13: 185-197, 2016.

9. Lu Z, Chouhan AK, Borycz JA, Lu Z, Rossano AJ, Brain KL, Zhou Y, Meinertzhagen IA and Macleod GT: High-probability neurotransmitter release sites represent an energy-efficient design. Curr Biol 26: 2562-2571, 2016.

10. Zhang $\mathrm{Y}$ and Bhavnani BR: Glutamate-induced apoptosis in neuronal cells is mediated via caspase-dependent and independent mechanisms involving calpain and caspase-3 proteases as well as apoptosis inducing factor (AIF) and this process is inhibited by equine estrogens. BMC Neurosci 7: 49, 2006.

11. Lamigeon C, Bellier JP, Sacchettoni S, Rujano $M$ and Jacquemont B: Enhanced neuronal protection from oxidative stress by coculture with glutamic acid decarboxylase-expressing astrocytes. J Neurochem 77: 598-606, 2001. 
12. Ye ZC and Sontheimer H: Astrocytes protect neurons from neurotoxic injury by serum glutamate. Glia 22: 237-248, 1998.

13. Ha JS, Dho SH, Youm TH, Kwon KS and Park SS: Astrocytic phospholipase A2 contributes to neuronal glutamate toxicity. Brain Res 1590: 97-106, 2014.

14. Negishi T, Ishii Y, Kawamura S, Kuroda Y and Yoshikawa Y: Cryopreservation of brain tissue for primary culture. Exp Anim 51: 383-390, 2002.

15. Haseleu J, Anlauf E, Blaess S, Endl E and Derouiche A: Studying subcellular detail in fixed astrocytes: Dissociation of morphologically intact glial cells (DIMIGs). Front Cell Neurosci 7: 54, 2013.

16. Zhou L, Li F, Xu HB, Luo CX, Wu HY, Zhu MM, Lu W, Ji X, Zhou QG and Zhu DY: Treatment of cerebral ischemia by disrupting ischemia-induced interaction of nNOS with PSD-95. Nat Med 16: 1439-1443, 2010.

17. Sun Y, März P, Otten U, Ge J and Rose-John S: The effect of gp130 stimulation on glutamate-induced excitotoxicity in primary hippocampal neurons. Biochem Biophys Res Commun 295 532-539, 2002

18. Bösel J, Gandor F, Harms C, Synowitz M, Harms U, Djoufack PC, Megow D, Dirnagl U, Hörtnagl H, Fink KB and Endres M: Neuroprotective effects of atorvastatin against glutamate-induced excitotoxicity in primary cortical neurones. J Neurochem 92: 1386-1398, 2005.

19. Jiang $\mathrm{Q}, \mathrm{Gu} Z$ and Zhang G: Activation, involvement and nuclear translocation of c-Jun $\mathrm{N}$-terminal protein kinase 1 and 2 in glutamate-induced apoptosis in cultured rat cortical neurons. Brain Res 956: 194-201, 2002.
20. Savaskan NE, Bräuer AU, Kühbacher M, Eyüpoglu IY, Kyriakopoulos A, Ninnemann O, Behne D and Nitsch R: Selenium deficiency increases susceptibility to glutamate-induced excitotoxicity. FASEB J 17: 112-114, 2003.

21. Maher P and Davis JB: The role of monoamine metabolism in oxidative glutamate toxicity. J Neurosci 16: 6394-6401, 1996.

22. Choi DW, Maulucci-Gedde M and Kriegstein AR: Glutamate neurotoxicity in cortical cell culture. J Neurosci 7: 357-368, 1987.

23. Amin N and Pearce B: Glutamate toxicity in neuron-enriched and neuron-astrocyte co-cultures: Effect of the glutamate uptake inhibitor L-trans-pyrrolidine-2,4-dicarboxylate. Neurochem Int 30: 271-276, 1997.

24. Hyndman AG: The effects of glutamate and kainate on cell proliferation in retinal cultures. Invest Ophthalmol Vis Sci 25: 558-563, 1984

25. Olney JW: Glutamate-induced neuronal necrosis in the infant mouse hypothalamus. An electron microscopic study. J Neuropathol Exp Neurol 30: 75-90, 1971.

26. Churn SB, Sombati S, Taft WC and DeLorenzo RJ: Excitotoxicity affects membrane potential and calmodulin kinase II activity in cultured rat cortical neurons. Stroke 24: 271-278, 1993.

This work is licensed under a Creative Commons

Attribution-NonCommercial-NoDerivatives 4.0

International (CC BY-NC-ND 4.0) License. 